\title{
Green Fuel Desulfurization with $\beta$-Cyclodextrin Aqueous Solution for Thiophenic Sulfides by Molecular Inclusion
}

\author{
Zunbin Duan ${ }^{a}$, He Bian ${ }^{a, b}$, Zhijian Gao ${ }^{a}$, Lijun Zhu ${ }^{a}$ and Daohong Xia*a \\ ${ }^{a}$ State Key Laboratory of Heavy Oil Processing, College of Chemical Engineering, China \\ University of Petroleum (East China), Qingdao 266580, China. \\ ${ }^{\mathrm{b}}$ Binzhou Key Laboratory of Material Chemistry, Department of Chemical Engineering and \\ Safety, Binzhou University, Binzhou 256603, China. \\ *Corresponding author. Tel.: +8653286981869; fax: +8653286981787. E-mail address: \\ xiadh@upc.edu.cn.
}

\section{List of Contents}

S1. Supplementary Figures.

Figure S1. Chemical structure and analytical configuration of $\beta$-cyclodextrin $(\beta \mathrm{CD})$.

Figure S2. Desulfurization performance of $\beta$-CD aqueous solution ( $\beta \mathrm{CD}$ aq) for n-octythiol.

Figure S3. Desulfurization performance of $\beta \mathrm{CD}$ aq and powder activated carbon for BT. 
Figure S4. UV absorption spectra and relation graphs of $1 / \triangle \mathrm{A}$ and $1 /[\beta \mathrm{CD}]_{0}$ of the thiophenic sulfide phosphate buffered solution with the $\beta \mathrm{CD}$ of different concentrations.

Figure S5. FT-IR spectra and XRD patterns of $\beta \mathrm{CD}$ and the three inclusion complexes.

Figure S6. SEM images of $\beta \mathrm{CD}, \beta \mathrm{CD}: \mathrm{T}, \beta \mathrm{CD}: \mathrm{BT}$, and $\beta \mathrm{CD}: \mathrm{DBT}$.

S2. Supplementary Tables.

Table S1. Molecular sizes of $\beta$-CD, T, BT, and DBT.

Table S2. Chemical shifts $(\delta)$ and the change of chemical shifts $(\triangle \delta)$ of T and $\beta C D$.

Table S3. Chemical shifts $(\delta)$ and the change of chemical shifts $(\triangle \delta)$ of BT and $\beta \mathrm{CD}$.

Table S4. Chemical shifts $(\delta)$ and the change of chemical shifts $(\triangle \delta)$ of DBT and $\beta \mathrm{CD}$.

Table S5. Possible paths for the formation of $\beta \mathrm{CD}$ :T by MD simulation.

Table S6. Possible paths for the formation of $\beta \mathrm{CD}$ :BT by MD simulation.

Table S7. Possible paths for the formation of $\beta C D$ :DBT by MD simulation.

S3. Supplementary Equations. 


\section{S1. Supplementary Figures.}

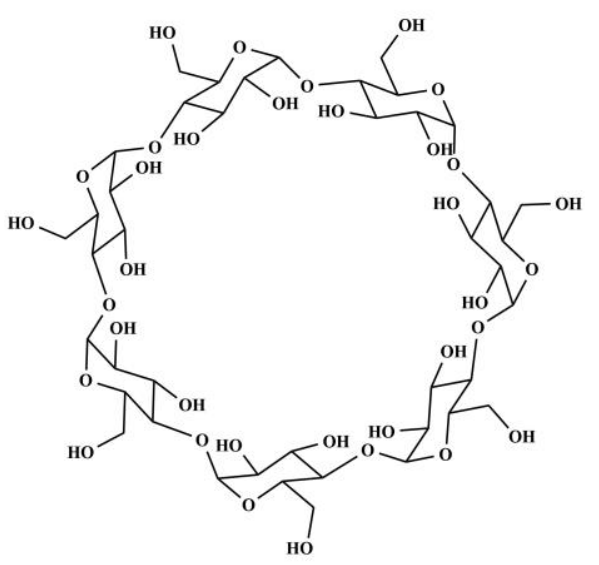

(a)

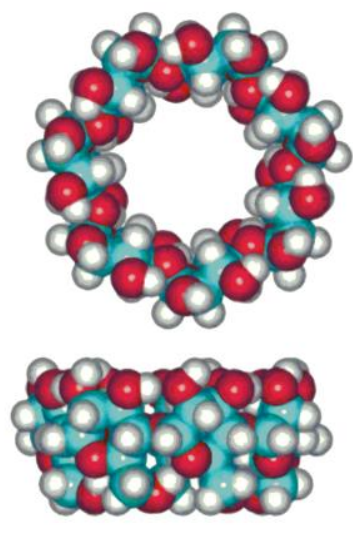

(b)

Figure S1. (a) Chemical structure and (b) analytical configuration of $\beta \mathrm{CD}$.

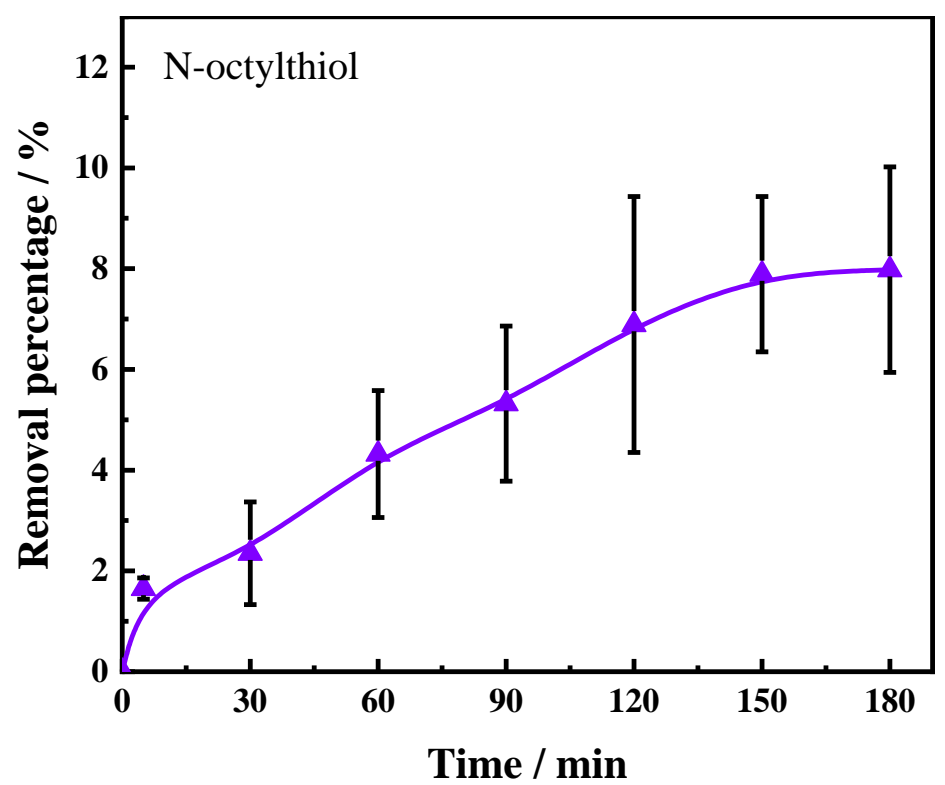

Figure S2. Desulfurization performance of $\beta C D$ aq for n-octythiol in n-heptane $(\beta C D$ mass content, 1.5 mass $\%$; temperature, $30{ }^{\circ} \mathrm{C} ; \beta \mathrm{CD}$ aq/n-heptane volume ratio, $\left.1: 1\right)$. 


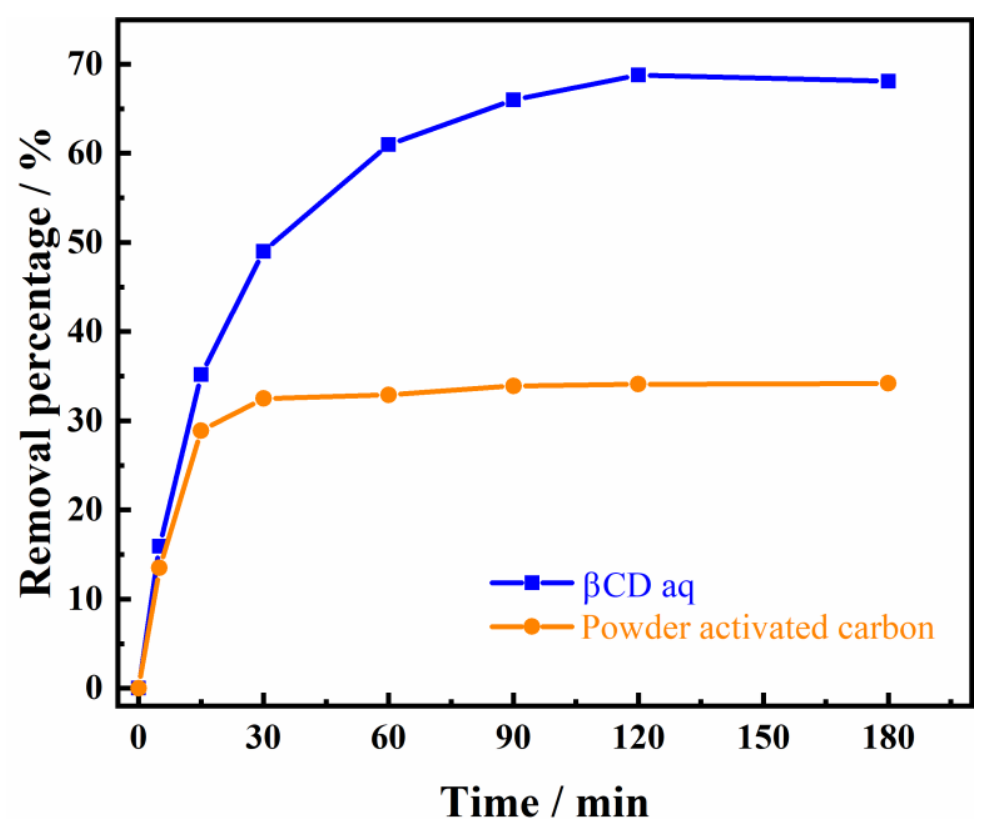

Figure S3. Desulfurization performance of $\beta \mathrm{CD}$ aq and powder activated carbon for $\mathrm{BT}$ in $\mathrm{n}$-heptane $\left(\beta \mathrm{CD}\right.$ mass content, 1.5 mass $\%$; temperature, $30^{\circ} \mathrm{C} ; \beta \mathrm{CD}$ aq/n-heptane volume ratio, 2: 1; powder activated carbon/n-heptane mass ratio, $1: 33$ ). 

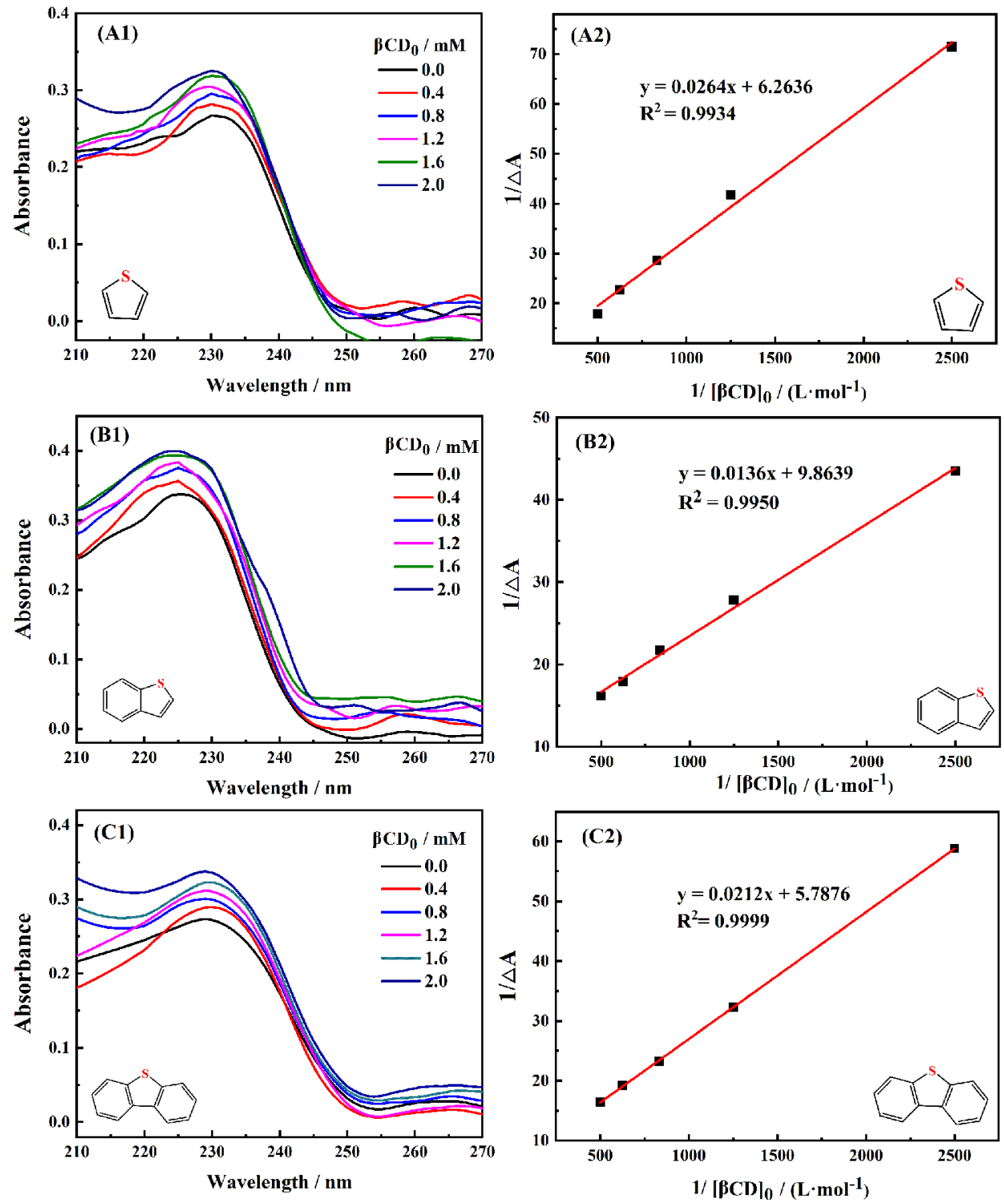

Figure S4. (A1, B1, C1) UV absorption spectra and (A2, B2, C2) relation graphs of 1/ $\triangle A$ and $1 /[\beta \mathrm{CD}]_{0}$ of the thiophenic sulfide phosphate buffered solution with the $\beta \mathrm{CD}$ of different contents. 

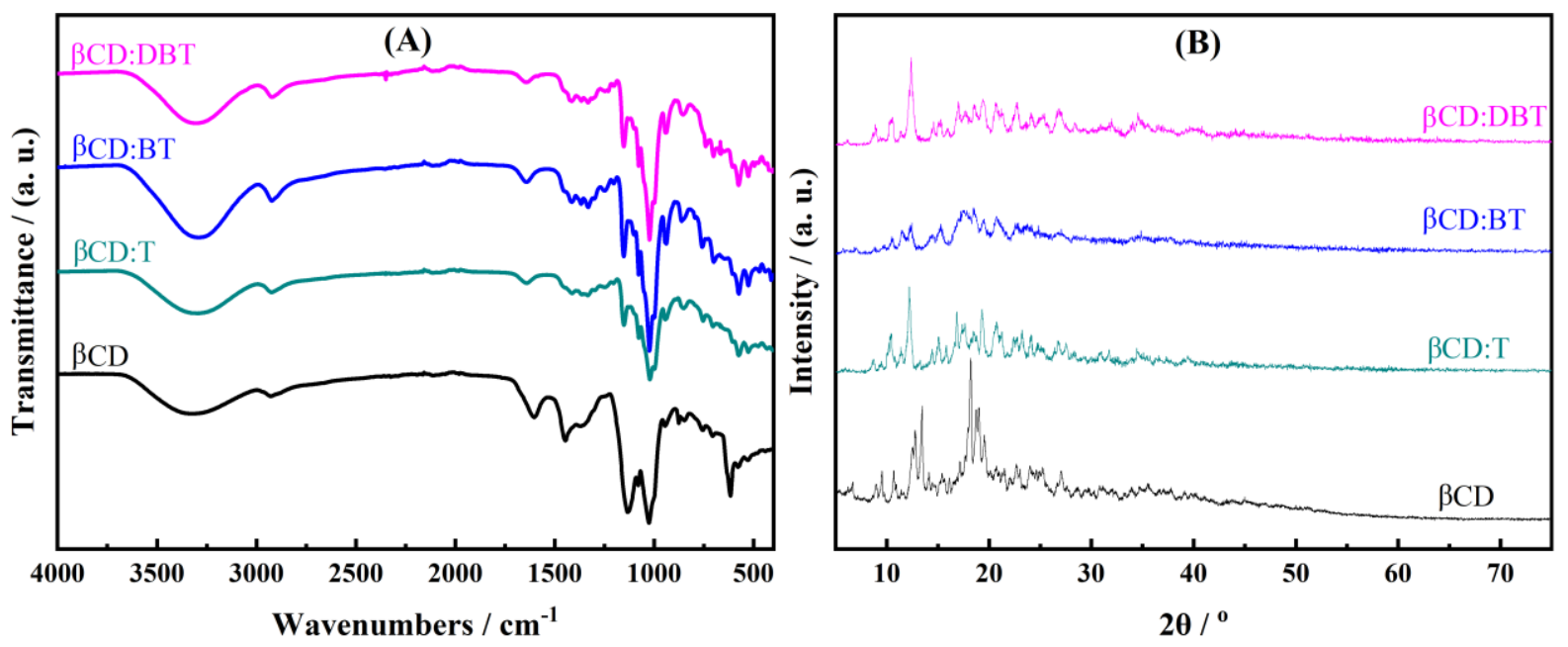

Figure S5. (A) FT-IR spectra and (B) XRD patterns of $\beta C D$ and the three inclusion complexes.

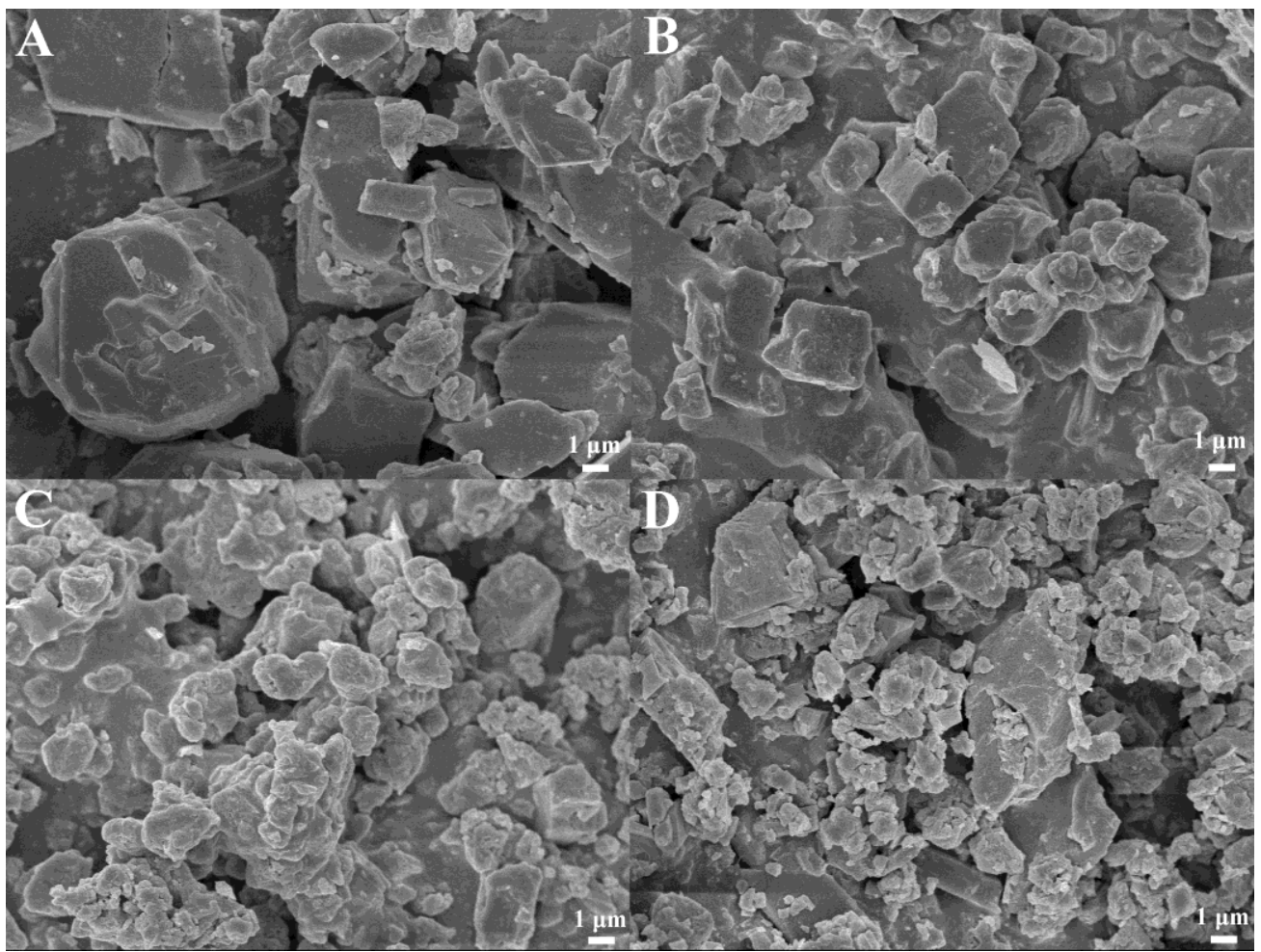

Figure S6. SEM images of (A) $\beta \mathrm{CD}$, (B) $\beta \mathrm{CD}$ : $\mathrm{T}$, (C) $\beta \mathrm{CD}$ : BT and (D) $\beta \mathrm{CD}: \mathrm{DBT}$. 


\section{S2. Supplementary Tables.}

Table S1. Molecular sizes of $\beta \mathrm{CD}$, T, BT and DBT.

\begin{tabular}{lll}
\hline Molecules & Length $/ \mathrm{nm}$ & Diameter $/ \mathrm{nm}$ \\
\hline$\beta \mathrm{CD}$ & $0.78-0.80$ & $0.60-0.65$ \\
$\mathrm{~T}$ & 0.34 & 0.45 \\
$\mathrm{BT}$ & 0.68 & 0.50 \\
$\mathrm{DBT}$ & 0.89 & 0.49 \\
\hline
\end{tabular}

Notes: the molecular sizes of T, BT and DBT were calculated by Chemoffice software.

Table S2. Chemical shifts $(\delta)$ and the change of chemical shifts $(\triangle \delta)$ of T and $\beta C D$.

\begin{tabular}{lllllll}
\hline \multirow{2}{*}{ Protons } & $\beta \mathrm{CD}$ & \multicolumn{5}{c}{$\mathrm{T}$} \\
\cline { 2 - 6 } & $\delta_{\beta \mathrm{CD} / \mathrm{ppm}}$ & $\delta \beta$ CD:T/ppm & $\Delta \delta$ & $\delta \mathrm{T} / \mathrm{ppm}$ & $\delta_{\beta \mathrm{CD}: \mathrm{T} / \mathrm{ppm}}$ & $\Delta \delta$ \\
\hline H-1(a, a') & 4.8319 & 4.8327 & 0.0008 & 7.5508 & 7.5681 & 0.0173 \\
H-2(b, b') & 3.3507 & 3.3507 & 0.0000 & 7.1519 & 7.1588 & 0.0069 \\
H-3 & 3.6603 & 3.6676 & 0.0073 & & & \\
H-4 & 3.2993 & 3.2995 & 0.0002 & & \\
H-5 & 3.5464 & 3.5513 & 0.0049 & & & \\
H-6 & 3.6305 & 3.6326 & 0.0021 & & \\
OH-2 & 5.7424 & 5.6976 & -0.0448 & & \\
OH-3 & 5.6829 & 5.6487 & -0.0342 & & \\
OH-6 & 4.4582 & 4.4317 & -0.0265 & & \\
\hline
\end{tabular}

Notes: $\triangle \delta=\delta_{\beta C D: T}-\delta_{\beta C D}$. 
Table S3. Chemical shifts $(\delta)$ and the change of chemical shifts $(\triangle \delta)$ of BT and $\beta \mathrm{CD}$.

\begin{tabular}{lllllll}
\hline \multirow{2}{*}{ Protons } & \multicolumn{7}{l}{ BCD } & \multicolumn{5}{c}{$\mathrm{BT}$} \\
\cline { 2 - 6 } & $\delta_{\beta \mathrm{CD}} / \mathrm{ppm}$ & $\delta_{\beta \mathrm{CD}: \mathrm{BT} / \mathrm{ppm}} \Delta \delta$ & $\delta_{\mathrm{BT}} / \mathrm{ppm}$ & $\delta_{\beta \mathrm{CD}: \mathrm{BT} / \mathrm{ppm}}$ & $\Delta \delta$ \\
\hline H-1(a) & 4.8319 & 4.8410 & 0.0091 & 7.7605 & 7.7692 & 0.0087 \\
H-2(b) & 3.3507 & 3.3594 & 0.0087 & 7.4700 & 7.4773 & 0.0073 \\
H-3(c) & 3.6603 & 3.6722 & 0.0119 & 7.8966 & 7.9021 & 0.0055 \\
H-4(d, e) & 3.2993 & 3.3078 & 0.0085 & 7.3754 & 7.3813 & 0.0059 \\
H-5(f) & 3.5464 & 3.5600 & 0.0136 & 8.0154 & 8.0225 & 0.0071 \\
H-6 & 3.6305 & 3.6423 & 0.0118 & & & \\
OH-2 & 5.7424 & 5.7173 & -0.0251 & & & \\
OH-3 & 5.6829 & 5.6656 & -0.0173 & & & \\
OH-6 & 4.4582 & 4.4345 & -0.0237 & & & \\
\hline
\end{tabular}

Notes: $\Delta \delta=\delta_{\beta C D: B T}-\delta_{\beta C D}$. 
Table S4. Chemical shifts $(\delta)$ and the change of chemical shifts $(\triangle \delta)$ of DBT and $\beta C D$.

\begin{tabular}{lllllll}
\hline \multirow{2}{*}{ Protons } & \multicolumn{5}{c}{ D CD } & \multicolumn{5}{c}{} \\
\cline { 2 - 6 } & $\delta_{\beta \text { CD } / \mathrm{ppm}}$ & $\delta_{\beta \text { CD:DBT }} / \mathrm{ppm}$ & $\Delta \delta$ & $\delta_{\mathrm{DBT}} / \mathrm{ppm}$ & $\delta_{\beta \text { CD:DBT }} / \mathrm{ppm}$ & $\Delta \delta$ \\
\hline H-1(a, a') & 4.8319 & 4.8337 & 0.0018 & 8.0418 & 8.0387 & -0.0037 \\
H-2(b, b') & 3.3507 & 3.3520 & 0.0013 & 7.5109 & 7.5089 & -0.0020 \\
H-3(c, c') & 3.6603 & 3.6707 & 0.0104 & 7.5338 & 7.5318 & -0.0020 \\
H-4(d, d') & 3.2993 & 3.2945 & -0.0048 & 8.3826 & 8.3811 & -0.0015 \\
H-5 & 3.5464 & 3.5548 & 0.0084 & & & \\
H-6 & 3.6305 & 3.6359 & 0.0054 & & & \\
OH-2 & 5.7424 & 5.7008 & -0.0416 & & & \\
OH-3 & 5.6829 & 5.6512 & -0.0317 & & & \\
OH-6 & 4.4582 & 4.4183 & -0.0401 & & & \\
\hline
\end{tabular}

Notes: $\Delta \delta=\delta_{\beta C D: D B T}-\delta_{\beta C D}$. 
Table S5. Possible paths for the formation of $\beta C D$ :T by MD simulation.

\begin{tabular}{|c|c|c|c|c|c|c|c|c|}
\hline $\begin{array}{l}\text { Simulation } \\
\text { times }\end{array}$ & $\begin{array}{l}\text { Formation } \\
\text { path }\end{array}$ & $\begin{array}{l}\text { Enter } \\
\text { time/ps }\end{array}$ & $\begin{array}{l}\text { Simulation } \\
\text { times }\end{array}$ & $\begin{array}{l}\text { Formation } \\
\text { path }\end{array}$ & $\begin{array}{l}\text { Enter } \\
\text { time/ps }\end{array}$ & $\begin{array}{l}\text { Simulation } \\
\text { times }\end{array}$ & $\begin{array}{l}\text { Formation } \\
\text { path }\end{array}$ & $\begin{array}{l}\text { Enter } \\
\text { time/ps }\end{array}$ \\
\hline 1 & 1 & 65 & 18 & 1 & 14 & 35 & 1 & 149 \\
\hline 2 & 2 & 201 & 19 & 2 & 998 & 36 & 2 & 514 \\
\hline 3 & 1 & 78 & 20 & 2 & 354 & 37 & 1 & 236 \\
\hline 4 & 1 & 241 & 21 & 1 & 198 & 38 & 2 & 117 \\
\hline 5 & 1 & 98 & 22 & 1 & 2231 & 39 & 1 & 769 \\
\hline 6 & 2 & 148 & 23 & 2 & 278 & 40 & 1 & 1138 \\
\hline 7 & 2 & 165 & 24 & 1 & 481 & 41 & 1 & 142 \\
\hline 8 & 1 & 142 & 25 & 1 & 147 & 42 & 2 & 348 \\
\hline 9 & 1 & 42 & 26 & 2 & 314 & 43 & 2 & 219 \\
\hline 10 & 1 & 1098 & 27 & 2 & 506 & 44 & 1 & 439 \\
\hline 11 & 1 & 398 & 28 & 2 & 29 & 45 & 1 & 29 \\
\hline 12 & 1 & 924 & 29 & 1 & 91 & 46 & 1 & 484 \\
\hline 13 & 2 & 89 & 30 & 1 & 263 & 47 & 1 & 75 \\
\hline 14 & 2 & 314 & 31 & 2 & 184 & 48 & 1 & 160 \\
\hline 15 & 1 & 261 & 32 & 1 & 96 & 49 & 2 & 178 \\
\hline 16 & 1 & 219 & 33 & 2 & 79 & 50 & 1 & 37 \\
\hline 17 & 1 & 23 & 34 & 1 & 108 & & & \\
\hline
\end{tabular}


Table S6. Possible paths for the formation of $\beta \mathrm{CD}$ :BT by MD simulation.

\begin{tabular}{|c|c|c|c|c|c|c|c|c|}
\hline Simulation & Formation & Enter & Simulation & Formation & Enter & Simulation & Formation & Enter \\
\hline times & path & time/ps & times & path & time/ps & times & path & time/ps \\
\hline 1 & 2 & 689 & 18 & 1 & 239 & 35 & 1 & 59 \\
\hline 2 & 1 & 1254 & 19 & 2 & 64 & 36 & 1 & 1435 \\
\hline 3 & 1 & 983 & 20 & 1 & 141 & 37 & 2 & 1583 \\
\hline 4 & 2 & 221 & 21 & 1 & 2491 & 38 & 1 & 324 \\
\hline 5 & 1 & 19 & 22 & 1 & 264 & 39 & 1 & 195 \\
\hline 6 & 1 & 359 & 23 & 2 & 291 & 40 & 1 & 1035 \\
\hline 7 & 2 & 1598 & 24 & 1 & 590 & 41 & 1 & 94 \\
\hline 8 & 1 & 3228 & 25 & 2 & 913 & 42 & 2 & 2981 \\
\hline 9 & 1 & 109 & 26 & 1 & 953 & 43 & 1 & 3814 \\
\hline 10 & 1 & 846 & 27 & 1 & 94 & 44 & 1 & 681 \\
\hline 11 & 2 & 1953 & 28 & 1 & 951 & 45 & 1 & 319 \\
\hline 12 & 1 & 184 & 29 & 2 & 184 & 46 & 2 & 2105 \\
\hline 13 & 1 & 751 & 30 & 1 & 953 & 47 & 1 & 73 \\
\hline 14 & 1 & 264 & 31 & 1 & 3211 & 48 & 1 & 329 \\
\hline 15 & 2 & 203 & 32 & 1 & 854 & 49 & 1 & 184 \\
\hline 16 & 1 & 1493 & 33 & 1 & 154 & 50 & 2 & 148 \\
\hline 17 & 1 & 1471 & 34 & 2 & 198 & & & \\
\hline
\end{tabular}


Table S7. Possible paths for the formation of $\beta \mathrm{CD}$ :DBT by MD simulation.

\begin{tabular}{|c|c|c|c|c|c|c|c|c|}
\hline Simulation & Formation & Enter & Simulation & Formation & Enter & Simulation & Formation & Enter \\
\hline times & path & time/ps & times & path & time/ps & times & path & time/ps \\
\hline 1 & 2 & 2766 & 18 & 1 & 166 & 35 & 1 & 965 \\
\hline 2 & 1 & 2316 & 19 & 2 & 1962 & 36 & 1 & 1952 \\
\hline 3 & 1 & 2909 & 20 & 1 & 607 & 37 & 1 & 309 \\
\hline 4 & 2 & 37 & 21 & 1 & 542 & 38 & 2 & 76 \\
\hline 5 & 1 & 2005 & 22 & 1 & 351 & 39 & 1 & 2984 \\
\hline 6 & 2 & 1154 & 23 & 1 & 39 & 40 & 1 & 328 \\
\hline 7 & 1 & 2766 & 24 & 2 & 98 & 41 & 1 & 961 \\
\hline 8 & 1 & 2004 & 25 & 2 & 584 & 42 & 1 & 1549 \\
\hline 9 & 1 & 26 & 26 & 1 & 3954 & 43 & 1 & 3209 \\
\hline 10 & 1 & 280 & 27 & 2 & 2148 & 44 & 1 & 601 \\
\hline 11 & 2 & 397 & 28 & 1 & 2984 & 45 & 2 & 2183 \\
\hline 12 & 1 & 2554 & 29 & 1 & 3961 & 46 & 2 & 940 \\
\hline 13 & 2 & 372 & 30 & 2 & 29 & 47 & 2 & 293 \\
\hline 14 & 1 & 2094 & 31 & 1 & 394 & 48 & 1 & 65 \\
\hline 15 & 1 & 2320 & 32 & 1 & 1965 & 49 & 1 & 481 \\
\hline 16 & 2 & 3516 & 33 & 1 & 2976 & 50 & 2 & 962 \\
\hline 17 & 1 & 60 & 34 & 1 & 1963 & & & \\
\hline
\end{tabular}




\section{S3. Supplementary Equations.}

\section{Equation of Benisi-Hildebrand.}

$$
\frac{1}{\Delta \mathrm{A}}=\frac{1}{\mathrm{Ka} \Delta \varepsilon_{\beta \mathrm{CD} \cdot \mathrm{s}}[\mathrm{S}]_{0}} \times \frac{1}{[\beta \mathrm{CD}]_{0}}+\frac{1}{\Delta \varepsilon_{\beta \mathrm{CD} \cdot \mathrm{s}}[\mathrm{S}]_{0}}
$$

where $\triangle \mathrm{A}$ is the difference in absorbance of sulfide in the presence and absence of $\beta \mathrm{CD}$; $\triangle \varepsilon_{\beta} \mathrm{CD} \cdot \mathrm{s}$ is the difference in the molar absorption coefficient of sulfide and the inclusion complex; $[\beta \mathrm{CD}]_{0}$ and $[\mathrm{S}]_{0}$ are the initial concentration of $\beta \mathrm{CD}$ and sulfide, respectively. 